\title{
Oficina digital: Experiência projetual para a Tulha da Fazenda do Pinhal
} Digital workshop: Project experience for the Tulha of Fazenda do Pinhal

\author{
Simone Helena Tanoue Vizioli \\ Instituto de Arquitetura e Urbanismo. USP. Brasil \\ simonehtv@sc.usp.br \\ Paulo César Castral \\ Instituto de Arquitetura e Urbanismo. USP. Brasil \\ pcastral@sc.usp.br
}

\author{
Joubert José Lancha \\ Instituto de Arquitetura e Urbanismo. USP. Brasil \\ joubertlancha@gmail.com
}

\begin{abstract}
The workshop was conceived as a laboratory of practices that articulate the exercise of the Project with the Digital Free Drawing to check the theoretical presuppositions of this research. It was intended to verify the limits of the analogical drawing in the digital platforms observing to directly influence of the drawing in the construction of the thought. The results allow us to point out that this is not a simple change of support but the possibility of a synergy between two logics of spellings, and the processes of cognition arising from such logics, in the teaching of Architecture Project.
\end{abstract}

Keywords: Digital free drawing; Pen display; Projective process; Graphic representation; Fazenda do Pinhal.

\section{Introdução}

Este artigo apresenta uma experiência de Oficina do Núcleo de Apoio à Pesquisa em Estudos de Linguagem em Arquitetura e Cidade (N.ELAC) do Instituto de Arquitetura e Urbanismo da Universidade de São Paulo (IAU.USP). O núcleo tem como objetivo discutir a aplicação do desenho à mão livre no processo projetivo arquitetônico e suas potencialidades por intermédio das plataformas digitais. A Oficina foi concebida como laboratório de práticas que entrelaçam o exercício do Projeto ao recurso do Desenho Livre Digital para aferição dos pressupostos teóricos e dos objetivos da pesquisa no processo de ensino-aprendizagem (edital MCTI/CNPq 14/2014). A atividade também fundamenta a ação institucional do IAU.USP de implementação do Laboratório de Desenho Livre Digital (edital Santander e-Grad 2015-2016) voltado para o ensino de arquitetura e urbanismo, com a abertura para os alunos do CAU.IAU em janeiro de 2018. A Oficina, realizada em outubro de $2016 \mathrm{com}$ a participação de docentes, pesquisadores e alunos da USP, da UNESP e do Politécnico de Milão, teve como proposta de projeto a intervenção na Tulha da Fazenda do Pinhal (São Carlos/SP/Br). Além do objetivo principal, a partir do contato direto com as evidências e manifestações da cultura local buscou-se levar os participantes a um processo ativo de conhecimento, apropriação e valorização da herança cultural do patrimônio (Horta, Grumberg, Monteiro, 1999).

No quadro histórico, o desenho foi se constituindo uma alternativa que ainda hoje subsiste. Hoje, vive-se uma época em que a informática e os meios de comunicação se renovam a cada instante, e neste percurso, também os meios de representação são palco de inovações tecnológicas que redesenham as alternativas de expressão. O meio digital permite que as possibilidades de sinergias entre ambas alternativas se preservem. (Seguí, 2007) (Veiga, Vizioli, 2015)
O presente artigo se propõe apresentar e discutir uma experiência em um dos lados desse processo atual, o desenho livre digital, inscrito nas primeiras etapas do desenvolvimento de um projeto, mais especificamente até a proposta conceitual.

\section{Sobre o Desenho Livre}

Para Herbert (1993) os desenhos de estudo são sempre incompletos e contingentes, estão entre um passado não resolvido e um futuro imprevisível. Também para Garner (2011), os esboços têm na ambiguidade um de seus fatoreschaves, isto porque, estas representações permitem novas possibilidades e também novas interpretações do projeto. Segundo Tversky (2002), os croquis são uma espécie de representação externalizada, usada como ferramenta cognitiva no processamento da memória e da informação. Maria Vasconcelos (2006) ressalta a possibilidade de se articular diferentes lógicas de pensamento por meio do desenho, ou seja, o gesto rápido permite numa mesma ação a constituição de relações objetivas e lineares e a percepção das mesmas de maneira integrada e generalizada.

Para Ginoulhiac (2009) a ambiguidade decorrente do baixo grau de definição de informação auxilia no processo de projeto entendido como um jogo de negociação entre os vestígios e índices de um pensamento passado e a busca por um sentido para uma solução em função das demandas que a proposta terá que atender. Nesse sentido o processo projetual apresenta uma relação dialética entre o investimento significante e a preservação da memória. Condição garantida pela imprecisão definida por Ginoulhiac. Pinheiro (2007) atenta que tal característica se deve pelo fato que a relação que 0 arquiteto estabelece com o desenho livre, próprio da fase de concepção de um projeto, se dá inscrita em processo topológico de busca de sentido. 


\section{Sobre 0 Desenho Livre Digital}

De acordo com Duff (2005, in VASCONCELOS, 2006) não é possível pensar o desenho hoje, da mesma forma que há trinta anos, pois existe uma nova gramática e sintaxe criada pelas novas tecnologias. A atividade de desenhar é dinâmica e reflete o contexto de seu tempo. Mas ressalta-se que o momento atual é de coexistência entre os diferentes tipos de tecnologias. É inquestionável o avanço que o $C A D$ imputou à área de Arquitetura, porém, de acordo com Righi (2008) estudos apontam que durante o processo de parametrização das soluções em sistemas $C A D$ ocorre uma simplificação digital da proposta inicial. Perde-se parte da imprecisão dos croquis, dos estudos, que permitem a flexibilização das ações dos arquitetos.

Herbert (1995) apontou as possibilidades criativas potencializadas pela sinergia entre os diferentes meios, analógico e digital, no desenvolvimento de projeto. Naquele momento tratava-se de rever o paradigma da relação entre o meio analógico e meio digital definida como one-way, isto é, primeiro o croqui sobre o papel e depois digitalizar as informações em um desenho assistido por computador. Hebert aponta nesse estudo a necessidade de se rever tal procedimento, explorando a complexidade de soluções gráficas capazes de sugerir novas formas para o projeto que um desenvolvimento menos linear possibilitaria.

Atualmente existem instrumentos que investem em sistemas de captura de movimento na busca de tornar tal interação o mais intuitiva possível e com uma precisão cada vez maior. Seja por superfície touch (tablets e mesas digitalizadoras), seja por canetas digitalizadoras (Inkling) e Spark da Wacom e Smart Writing Set da Moleskine) o desenho livre passa a ser incorporado como uma alternativa real aos outros processos associados ao desenvolvimento de projeto no meio digital. Anna Kenoff (2017), da Morpholio, atenta que esse processo conta agora com desenvolvimento de softwares voltados para arquitetos pelas grandes empresas como Adobe, Autodesk, Concepts e a própria Morpholio.

Inaugura-se a possibilidade de atualizar o conceito de paperless studio (Norman, 2001), que teve como marco experiência piloto implantada, em 1994, por Bernard Tschumi fruto da atuação na Columbia's Graduate School of Architecture, Planning, and Preservation.

\section{Sobre a Oficina de Desenho Livre Digital}

A oficina consistiu em uma atividade projetual desenvolvida por 05 equipes: 04 alunos de do IAU.USP de diversos períodos (atelier vertical); 01 graduado como tutor. Cada equipe contou com o acompanhamento de 02 professores do IAU e dois professores convidados (um do Politécnico de Milão e outro da UNESP). O N.ELAC mobilizou os seguintes equipamentos: 24 IPads; 05 tablets Inote Sansung; 02 mesas digitalizadoras Cintiq 24HD; 04 mesas digitalizadoras Cintiq 27HD; 02 projetores multimídia. Tais equipamentos configurarão o que será o Laboratório de Desenho Livre Digital, montado em caráter experimental em um dos ateliers do IAU.USP.

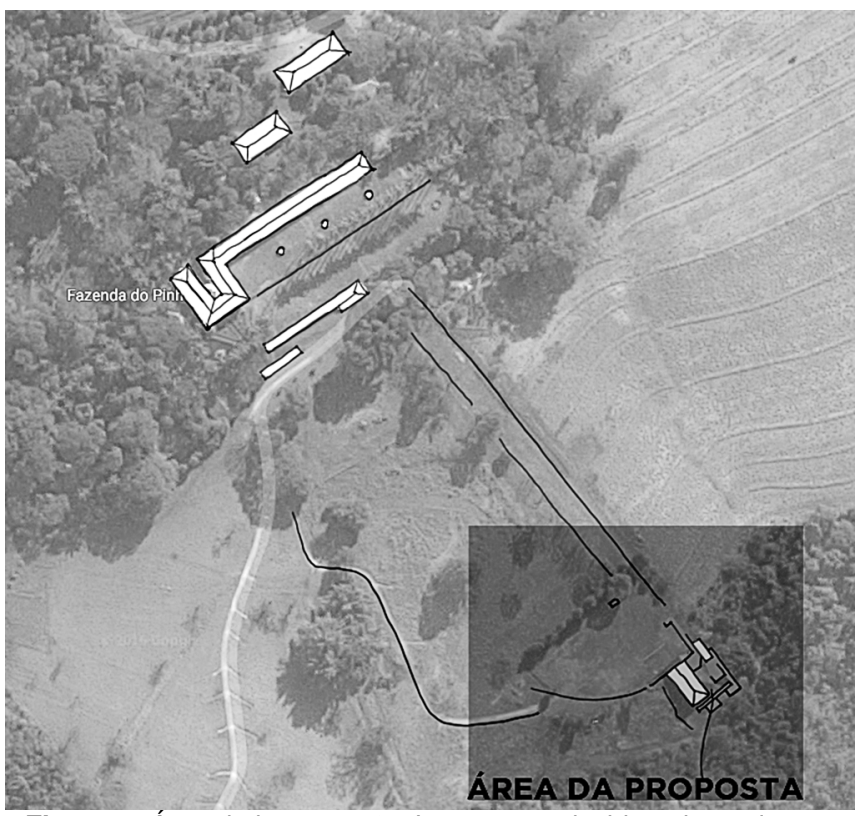

Figura 1: Área de intervenção. Imagem produzida pela equipe 02.

As atividades desta oficina se desenvolveram em dois momentos: análise e proposição. A análise consistiu em uma visita, de um dia, à área objeto para a produção de desenhos de observação à mão livre no meio digital (em tablets Samsung e Ipad), com o objetivo de contrapor olhares reversos e complementares sobre o objeto de intervenção, focados nas seguintes questões: aproximação ao lugar I: produção de uma série de desenhos que mostram a relação da área-objeto com a paisagem (Figura 1), leitura da Fazenda como um todo; aproximação ao lugar II: a percepção dos aspectos patrimoniais e construtivos; e aproximação ao lugar III: a percepção fenomênica da área-objeto, através das possibilidades atuais e potenciais dos espaços da Tulha e as relações entre o interior e exterior (Figura 2).

A proposição, desenvolvida em dois dias no IAU, tinha como problema a adequação da edificação, que serviu como processamento e armazenamento do grão de café, para atender um programa de educação patrimonial como suporte às visitas monitoradas da fazenda.

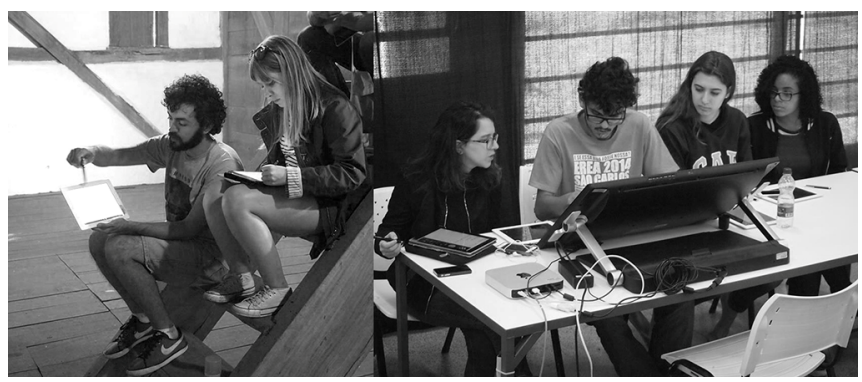

Figura 2: Equipes durante a análise (esq.) e proposição (dir.). Foto acervo da pesquisa. 
Cada equipe teve que se posicionar em relação às funcionais e às patrimoniais. As propostas, de um modo geral, tomaram partido da situação topográfica, oriunda da lógica produtiva do transporte do grão de café, e do enquadramento da paisagem natural, mas apresentaram soluções diferenciadas na ocupação do sítio sendo que: duas propostas criam um percurso qualificado sobre as ruínas da Roda D’Água, duas propostas definem anexos a uma certa distância da edificação principal, e uma última replica o volume da edificação.

O processo de projeto foi realizado por meio de redesenhos livres e intervenções gráficas sobre imagens do local e da documentação projetual, utilizando tablets para as proposições individuais e mesas digitalizadoras (cintiq) para as proposições coletivas. Adotou-se os programas Adobe Photoshop e Autodesk SketchBook.

A oficina representa a quarta ação de pesquisa do N.ELAC: IAU.USP-São Carlos; Ouro Preto-MG e Porto-Portugal. A partir dos estudos de Gero, Lee e Tang (2010), interessou à pesquisa desenvolvida nessas quatro oficinas adotar os protocolos simultâneos e os retrospectivos (Concurrent protocols e Retrospective protocols) que se caracterizam pela articulação entre a coleta de dados durante o processo, através de registros gráficos da ação projetual, e a entrevista com os agentes criadores após a conclusão da atividade.

É importante destacar que a pesquisa parametrizou a observação atentando para limites éticos e informou todos os envolvidos que tal observação estaria ocorrendo. Objetivou-se a diminuição das questões características desse tipo de pesquisa qualitativa que podem a comprometer a integridade dos resultados (Eisner, 1998)

A garantia de equidade de desempenho foi uma das estratégias adotadas pela pesquisa. A experiência com os mesmos programas e composição de equipes verticais (alunos dos diversos períodos do curso) para suprir carências de habilidades foram previstas e planejadas. Nesse sentido as considerações a seguir abdicam da apresentação de tabulações quantitativas para inferir sobre os resultados observados.

\section{Sobre as Grafias}

Nas primeiras aproximações ao objeto de intervenção foram realizados os levantamentos e desenhos com as tablets. Os alunos tomaram partido da vantagem desse meio permitir o armazenamento ativo dos desenhos realizados no local, ou seja, eles puderam, a qualquer momento, convocar as imagens produzidas e editá-las, no mesmo software em que foram elaboradas ou por meio de outros softwares que possibilitem trabalhar com ferramentas diferentes. Levantamento e projeto se misturam, processo que não é novo ou diferente do usual, a dinâmica é que se alterou, já que durante os primeiros desenhos de levantamento do sítio e do edifício, alternativas de projeto podem ser verificadas através de camadas sobrepostas aos desenhos e fotos. $O$ fato de estar diante do edifício, construindo um levantamento/proposição potencializa o processo de checagem e simultânea reflexão (Figura 3).

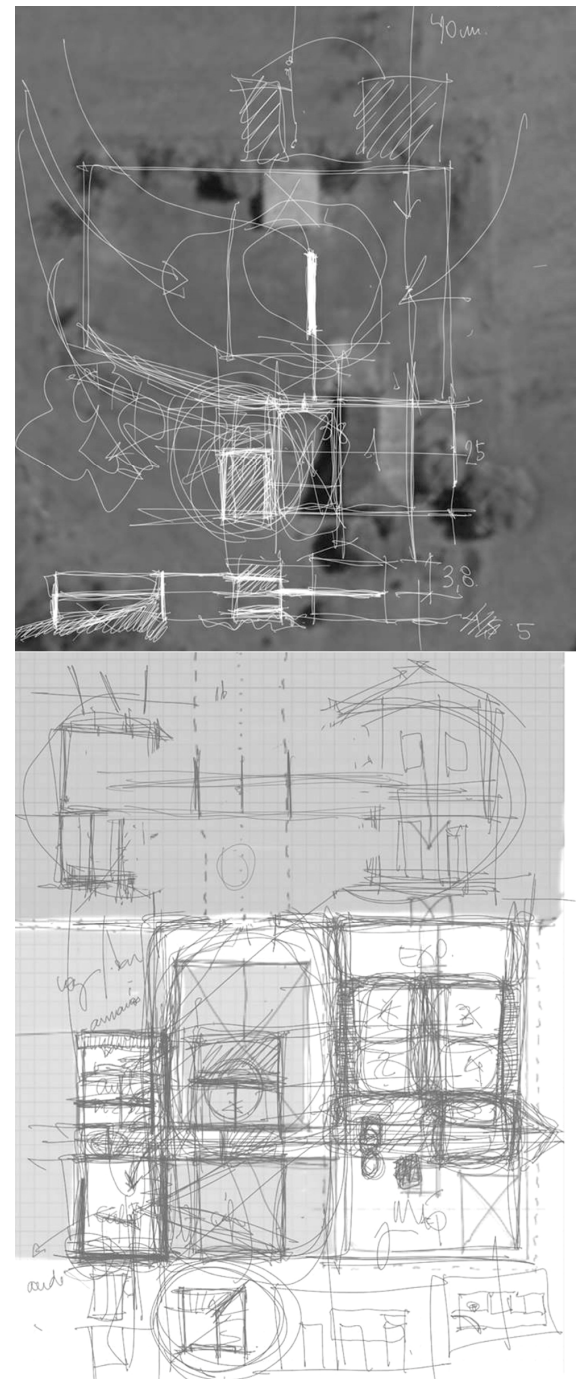

Figura 3: Peças gráficas produzidas pela equipe 04.

O recurso do desenho em camadas (layers), originalmente, destina-se a organizar as informações para um fácil acesso na edição e para definir as qualidades gráficas na saída impressa do arquivo. Durante a oficina, os alunos utilizaram tal recurso também para organizar os estudos, mas lançaram mão de outros parâmetros próprios do processo projetivo: as diversas propostas eram separadas em camadas que poderiam ser ativadas a título de comparação. Utilizaram esse recurso também para realizar uma análise das propostas realizadas bem como sobrepor a tais análises novas propostas (Figura 4). 


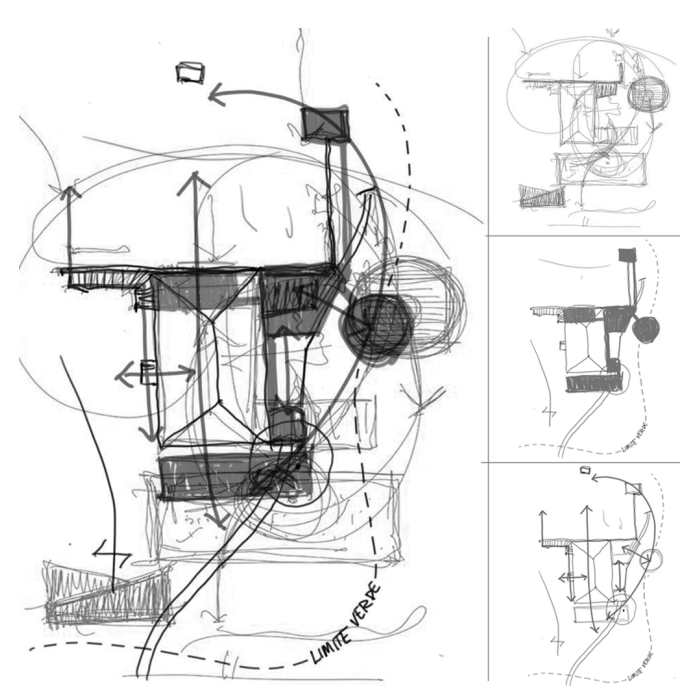

Figura 4: Uso de Camadas. Imagem produzida pela equipe 02.

Observou-se, nesse novo uso das camadas, um processo mais dinâmico do que os processos próprios ao uso de um meio exclusivo. No meio analógico, as diversas propostas tendem a se apagarem com a sobreposição dos traços ou pela baixa transparência dos papéis utilizados. Na oficina, os alunos puderam transitar entre os vários momentos do processo com uma facilidade que possibilitou ativar a relação dialética entre a memória dos registros e a busca de novas formas.

Cada equipe contava com uma mesa digitalizadora (cintiq) e cada membro tinha em mãos uma tablet para produção individual a ser compartilhada em rede. Nas mesas digitalizadoras foram realizados os desenhos coletivos em projeção ortogonal (plantas, cortes e elevações) tendo como base as imagens da documentação de projeto (não foram utilizados os arquivos .dwg) e imagens de satélite (google maps). Nas tablets também foram realizados tais desenhos, mas os alunos também experimentaram desenhos em perspectiva livre (realizada sem o uso de plantas) a partir das fotos que realizaram no local. A diferença entre os usos do equipamento se deu em função, segundo depoimentos, das características de cada desenho. Nas discussões em grupo os desenhos sobre plantas e cortes permitiam um registro mais rápido das opções projetivas, enquanto que a intervenção em fotografias necessitava de uma atenção maior do desenhador (Figura 5).

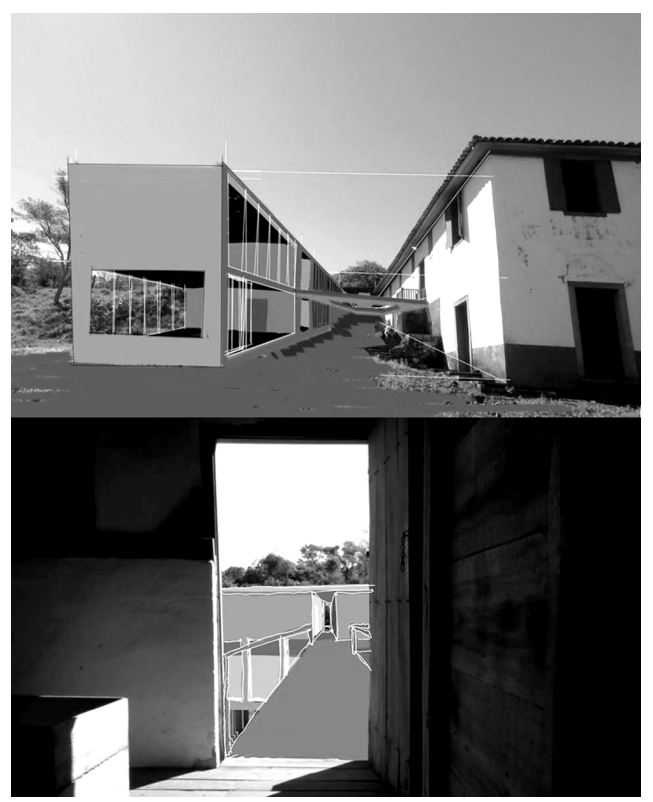

Figura 5: Desenho livre digital sobre fotografia Imagem produzida pela equipe 04 .

O início dos trabalhos foi marcado pelo uso de diagramas e desenhos sobre plantas e cortes. $\mathrm{Na}$ medida que se familiarizavam com a ação do desenhar utilizando os tablets, os alunos experienciaram pensar soluções projetuais a partir da ação sobre fotografias, simulando relações volumétricas e espaciais. Os alunos registraram verbalmente a preferência pelos desenhos sobre fotografia como uma forma mais adequada para o desenvolvimento de ideias iniciais em comparação com as diversas etapas necessárias aos outros programas de modelagem digital 3D. (Figura 6).

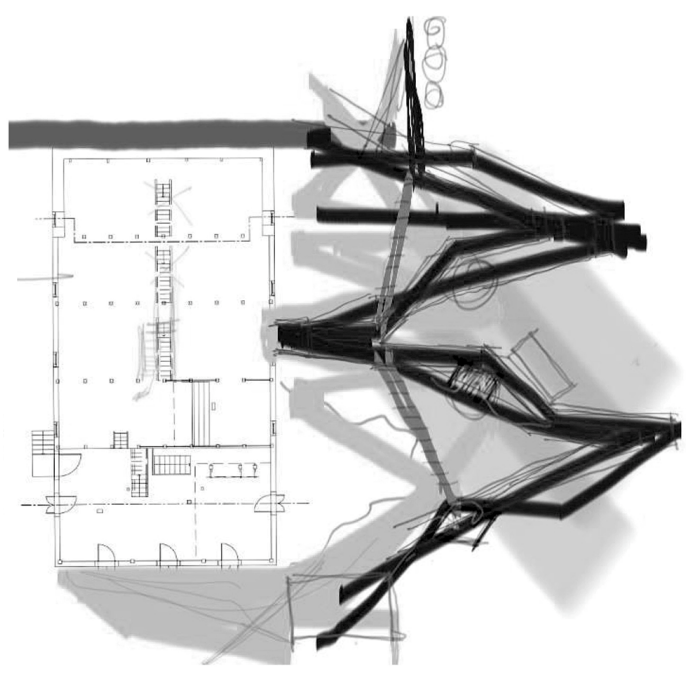

Figura 6: Desenho livre digital sobre planta. Imagem produzida pela equipe 05 . 
As linhas geralmente se caracterizavam no início do desenvolvimento de projeto pela sua fatura sem a concorrência de instrumentos técnicos (réguas ou programas $C A D$ ), que iriam comparecer quando a proposta adquirisse contornos mais precisos. O início das proposições ocorreram de forma semelhante, mas a adoção de linhas precisas, devido aos recursos dos programas adotados, compareceu bem antes da definição com maior clareza da proposta.

Observou-se a experimentações de outras situações: a) o uso de fotografias (fotos do local ou imagens de satélite), colocou, desde o princípio da ação projetual, uma relação topológica entre as linhas e as texturas da imagem; b) o uso de recursos gráficos de preenchimentos (pincéis e marcadores) sem a concorrência de limites pré-estabelecidos que tanto o Adobe Photoshop quanto o Autodesk SketchBook permitem. Nesse sentido foi possível experimentar a busca de formas para a proposta por meio da articulação de superfícies, passando a função da linha de propositiva para ênfase ao destacar a opção da equipe. Uma inversão cognitiva no processo de representação.

Por meio das tablets e mesas digitalizadoras somadas aos programas adotados facilitou-se somar e subtrair ideias por meio de alterações nos desenhos de forma interativa. $O$ uso dos recursos cognitivos dos meios de representação (Chiarella, 2009) puderam ser experienciados na mudança de atitude pelos alunos envolvidos na ação projetual.

\section{Considerações}

As intervenções gráficas sobre a documentação gráfica da edificação existente, nas atividades da presente oficina, revelam índices de possíveis questões a serem analisadas relativas ao uso desse ambiente de desenho livre digital para as fases posteriores do desenvolvimento de projeto. Verificar a permanência dessas ações de sinergias articuladas com os produtos do uso de programas de desenho numérico se coloca para a presente pesquisa como um universo necessário de continuidade. Por fim, é importante apontar que a passagem do desenho à mão livre analógico para o desenho livre digital, no presente caso estudado, não se configurou como uma simples alteração de suporte, do papel à tela do tablet, mas representou a possibilidade de uma sinergia entre duas lógicas de grafia, entre duas linguagens. Assim o processo de sistematização próprio dos softwares pôde ser contagiado pelo imediatismo do desenho à mão livre, e este ter seu processo de reconstrução significante potencializado por meio das características digitais de geração de informação e sua posterior edição.

\section{Agradecimentos}

Agradecemos às pessoas envolvidas na realização da oficina (pesquisadores, alunos e técnicos), ao IAU.USP pelo suporte e às agências de fomento CNPq, Santander (e-Grad) e Fapesp pelo apoio.

\section{Referências}

Chiarella, M. (2009). Unfolding Architecture. Laboratorio de Representación e Ideación. Tesis doctoral. Universidad Politécnica de Catalunya, Barcelona.

Eisner, E. W. (1998). El ojo ilustrado. Indagación cualitativa y mejora de la práctica educativa. Barcelona: Paidós Educador.

Garner, S. (2001). Comparing graphic actions between remote and proximal design teams. Design Studies, Vol. 22.

Gero, J. S.; Lee, Y. Y.; Tang, H. H. (2011) Comparing collaborative colocated and distributed design processes in digital and traditional sketching environments: A protocol study using the functionbehaviour-structure coding scheme. Design Studies, 32 (2011) (pp. 1-29)

Ginoulhiac, M. (2009). A interpretação da representação como condição disciplinar para o projeto de arquitetura. Arquitextos, São Paulo, ano 09, n.107.00, Vitruvius, abril. http:// www.vitruvius.com.br/revistas/read/arquitextos/09.107/55

Herbert, D. M. (1993). Architectural Study Drawings. Van Nostrand Reinhold. New York.

Herbert, D. M. (1995) "Models, Scanners, Pencils, and CAD: Interactions Between Manual and Digital Media." In: Computing in Design - Enabling, Capturing and Sharing Ideas: ACADIA Conference Proceedings, 21-34. ACADIA. Seattle, Washington: University of Washington

Horta, M. de L. P., Grumberg, E., \& Monteiro, A. Q. (1999). Guia Básico da Educação Patrimonial. Brasília: Instituto do Patrimônio Artístico Nacional, Museu Imperial.

Kenoff, Anna. (2017) "Décadas depois do surgimento do CAD, a arquitetura está se livrando do papel - desta vez é sério" [Decades After the Rise of CAD, Architecture Is Going "Paperless"-For Real This Time] 21 Jul 2017. ArchDaily Brasil. (Trad. Santiago Pedrotti, Gabriel) Acessado 21 Jul 2017. <http://www.archdaily.com.br/br/875901/decadas-depois-dosurgimento-do-cad-a-arquitetura-esta-se-livrando-do-papeldesta-vez-e-serio>

Norman, D. A. (2001) Towards a Paperless Studio, Work in Progress; ACADIA 2001 Pp: 336-341

Pinheiro, V.M.(2007). O Esquisso como lugar de convergência. in: Revista Lusófona de Arquitectura e Educação. Lisboa: Laboratório de Arquitectura (LABART) da Universidade Lusófona de Humanidades e Tecnologias (pp. 59-69).

Veiga, J.; Vizioli, S.H.T. (2015) Processo criativo digital: do croqui à impressão 3D Digital creative process: from sketching to 3D printing sigradi. In: Anais Congresso Iberoamericano de gráfica digital SIGRADI, Florianópolis.

Righi, T. A .F; Celani, G. (2008). Esboços na era digital: uma discussão sobre as mudanças na metodologia de projeto arquitetônico. In Anais Congresso Iberoamericano de gráfica digital SIGRADI, Havana, Cuba.

Seguí, J. (2007). Edificación, arquitectura, enseñanza de La arquitectura, modelización y dibujo. Revista EGA expresión gráfica arquitectónica, Vol. 12.

Tversky, B. (2002). What do sketches say about thinking? Department of Psychology Stanford University Stanford, CA.

Vasconcelos, H. E. M. (2006). Questioning drawing for designers: Project work as a strategy and examples from practice. In 2006 Desining Research Society. International Conference in Lisbon. IADE. 